\title{
MOSFIRE, the Multi-Object Spectrometer For Infra-Red Exploration at the Keck Observatory
}

\author{
Ian S. McLean*a, Charles C. Steidel ${ }^{\mathrm{b}}$, Harland Epps ${ }^{\mathrm{c}}$, Nicholas Konidaris ${ }^{\mathrm{b}}$, Keith Matthews ${ }^{\mathrm{b}}$, Sean \\ Adkins $^{\mathrm{d}}$, Ted Aliado ${ }^{\mathrm{a}}$, George Brims ${ }^{\mathrm{a}}$, John Canfield ${ }^{\mathrm{a}}$, John Cromer ${ }^{\mathrm{b}}$, Jason Fucik ${ }^{\mathrm{b}}$, Kristin Kulas ${ }^{\mathrm{a}}$, \\ Gregory Mace $^{\mathrm{a}}$, Ken Magnone ${ }^{\mathrm{a}}$, Hector Rodriguez ${ }^{\mathrm{b}}$, Gwen Rudie ${ }^{\mathrm{b}}$, Ryan Trainor ${ }^{\mathrm{b}}$, Eric Wang ${ }^{\mathrm{a}}$ Bob \\ Weber $^{\mathrm{b}}$ and Jason Weiss ${ }^{\mathrm{a}}$. \\ ${ }^{a}$ University of California, Los Angeles, CA, USA 90095-1547; \\ ${ }^{\mathrm{b}}$ California Institute of Technology, Pasadena, CA, USA 91125; \\ ${ }^{\mathrm{C}}$ University of California, Santa Cruz, CA, USA 95064; \\ ${ }^{\mathrm{d}}$ W. M. Keck Observatory, Waimea, HI, USA 96743
}

\begin{abstract}
This paper describes the as-built performance of MOSFIRE, the multi-object spectrometer and imager for the Cassegrain focus of the 10-m Keck 1 telescope. MOSFIRE provides near-infrared $(0.97$ to $2.41 \mu \mathrm{m})$ multi-object spectroscopy over a 6.1' x 6.1' field of view with a resolving power of $\mathrm{R} \sim 3,500$ for a $0.7^{\prime \prime}(0.508 \mathrm{~mm})$ slit $(2.9$ pixels in the dispersion direction), or imaging over a field of view of $\sim 6.9^{\prime}$ diameter with $\sim 0.18^{\prime \prime}$ per pixel sampling. A single diffraction grating can be set at two fixed angles, and order-sorting filters provide spectra that cover the K, H, J or Y bands by selecting $3^{\text {rd }}$, $4^{\text {th }}, 5^{\text {th }}$ or $6^{\text {th }}$ order respectively. A folding flat following the field lens is equipped with piezo transducers to provide tip/tilt control for flexure compensation at the $<0.1$ pixel level. Instead of fabricated focal plane masks requiring frequent cryo-cycling of the instrument, MOSFIRE is equipped with a cryogenic Configurable Slit Unit (CSU) developed in collaboration with the Swiss Center for Electronics and Microtechnology (CSEM). Under remote control the CSU can form masks containing up to 46 slits with $\sim 0.007-0.014 "$ precision. Reconfiguration time is $<6$ minutes. Slits are formed by moving opposable bars from both sides of the focal plane. An individual slit has a length of 7.0" but bar positions can be aligned to make longer slits in increments of 7.5". When masking bars are retracted from the field of view and the grating is changed to a mirror, MOSFIRE becomes a wide-field imager. The detector is a $2 \mathrm{~K} \times 2 \mathrm{~K} \mathrm{H} 2-\mathrm{RG} \mathrm{HgCdTe}$ array from Teledyne Imaging Sensors with low dark current and low noise. Results from integration and commissioning are presented.
\end{abstract}

Keywords: spectrometer, near-infrared, multi-object, Configurable Slit Unit, MOSFIRE, Keck Observatory

\section{INTRODUCTION}

MOSFIRE is a third generation instrument for the W. M. Keck Observatory (WMKO) ${ }^{1}$. Its requirements, design and development up until first light in the laboratory on July 1, 2010 were described in a previous paper ${ }^{2}$. MOSFIRE was delivered to the W. M. Keck Observatory on February 8, 2012 and "first light" on the Keck 1 telescope was obtained on April 4, 2012. This paper describes the performance of the as-built instrument based on early commissioning results. A multi-object spectrograph and wide-field camera for the near-infrared (0.97 to $2.41 \mu \mathrm{m})$, MOSFIRE's special feature is the cryogenic Configurable Slit Unit (CSU) that is reconfigurable under remote control in less than 6 minutes without any thermal cycling. Bars move in from each side to form up to 46 short slits. When the bars are removed MOSFIRE becomes a wide-field imager. The instrument was developed by teams from the University of California, Los Angeles (UCLA), the California Institute of Technology (CIT) and the University of California, Santa Cruz, (UCSC). The CoPrincipal Investigators are Ian S. McLean (UCLA) and Charles C. Steidel (CIT), and the project was managed by WMKO Instrument Program Manager, Sean Adkins. MOSFIRE was funded in part by the Telescope System Instrumentation Program (TSIP), which is operated by AURA and funded by the National Science Foundation, and by a private donation to WMKO by Gordon and Betty Moore.

*mclean@astro.ucla.edu

Ground-based and Airborne Instrumentation for Astronomy IV, edited by lan S. McLean, Suzanne K. Ramsay, Hideki Takami, Proc. of SPIE Vol. 8446, 84460J · ( ) 2012 SPIE · CCC code: 0277-786X/12/\$18 · doi: 10.1117/12.924794 


\section{SUMMARY OF THE AS-BUILT DESIGN}

Top level requirements for MOSFIRE were presented in an earlier paper ${ }^{2}$ and details of MOSFIRE's design reviews are available on our public web site (http://irlab.astro.ucla.edu/mosfire/index.html). Several technical challenges were encountered following first light in the lab during the fifth cool down. All problems were resolved without de-scopes and MOSFIRE was shown to meet all of its design requirements. Among MOSFIRE's key features are the following. It is a large vacuum-cryogenic instrument mounted in a rotating module at the Keck 1 Cassegrain focus. There is a heated double entrance window to prevent condensation, a unique configurable slit unit (CSU) operating over an aperture of $267 \times 267 \mathrm{~mm}$, a tip-tilt flexure compensation system, a large double filter wheel, a rotating/tracking pupil stop, and a mirror/grating turret. The detector is a Teledyne $2 \mathrm{~K} \times 2 \mathrm{~K}$ H2-RG plus SIDECAR ASIC 3 . MOSFIRE's optical system was designed by Harland Epps (UCSC) and the optical system was aligned and assembled using UCLA's Coordinate Measuring Machine (CMM) under the leadership of Nick Konidaris ${ }^{4}$. The CSU was designed and developed by CSEM, the Swiss Center for Electronics and Microtechnology ${ }^{5}$. MOSFIRE was shipped to the Observatory after its tenth cool down in the lab. This section reports on integration and testing in the lab.

Measurements using arc lamp spectra show that the wavelength coverage for spectroscopy spans 0.97 to $2.41 \mu \mathrm{m}$ with a resolving power of $\mathrm{R}=3,380,3,310,3,660$ and 3,620 in Y, J, H and $\mathrm{K}$ bands respectively, for a 0.7 " (2.9 pixel) slit, which is slightly better than the specification. With custom order-sorting filters, the same diffraction grating is used in four orders $(6,5,4$, and 3$)$ to cover the bands $\mathrm{Y}, \mathrm{J}, \mathrm{H}$, and $\mathrm{K}$ as required. To optimize the location of the spectra on the $2 \mathrm{~K} \times 2 \mathrm{~K}$ HgCdTe Hawaii 2-RG (H2-RG) detector two fixed and repeatable grating positions are used, one for $\mathrm{Y}$ and $\mathrm{J}$ and the other for $\mathrm{H}$ and $\mathrm{K}$. By driving against fixed stops these grating positions are repeatable to 0.1 pixels or better. Spectra are aligned along detector rows to better than $0.08^{\circ}$. A mirror mounted back-to-back with the grating enables MOSFIRE to change from spectroscopy to imaging mode. The desired pixel scale for imaging was 0.180 " and this has been achieved; the measured value is 0.1798". MOSFIRE's optical design also gives the desired field of view. For imaging this is a 6.9' diameter field projected onto the active detector area ( $2040 \times 2040$ pixels) which therefore covers $6.113^{\prime} \mathrm{x} 6.113^{\prime}$. Slits can be placed anywhere in the field but are optimally positioned in the central 6.1' x 3.0' region. The cryogenic configurable slit unit (CSU) provides 46 slits, using 92 masking bars which can be moved across the entire field of view. Each bar has an infrared-black knife edge. Slits are 7.01" long and the masking bars used to create the slits have an overlap region of $0.96 "$ to minimize light leakage. When a longer slit is formed using two pairs of bars, the total length is $14.98 "(2 \times 7.01 "+0.96 ")$. Measurements show that a full re-configuration of a slit mask takes less than six minutes. Detailed testing shows that the slits are extremely repeatable irrespective of orientation $(\sim 6 \mu \mathrm{m}$ in the CSU plane or $<0.02$ " on sky). Moreover, the flexure compensation system (FCS) maintains the position of images at the detector to better than 0.1 pixels rms for all position angles and all elevation angles greater than $22^{\circ}$ (air mass $=2.67$ ). Correction is based on a model which only requires the elevation angle of the telescope and position angle (PA) of the instrument rotator.
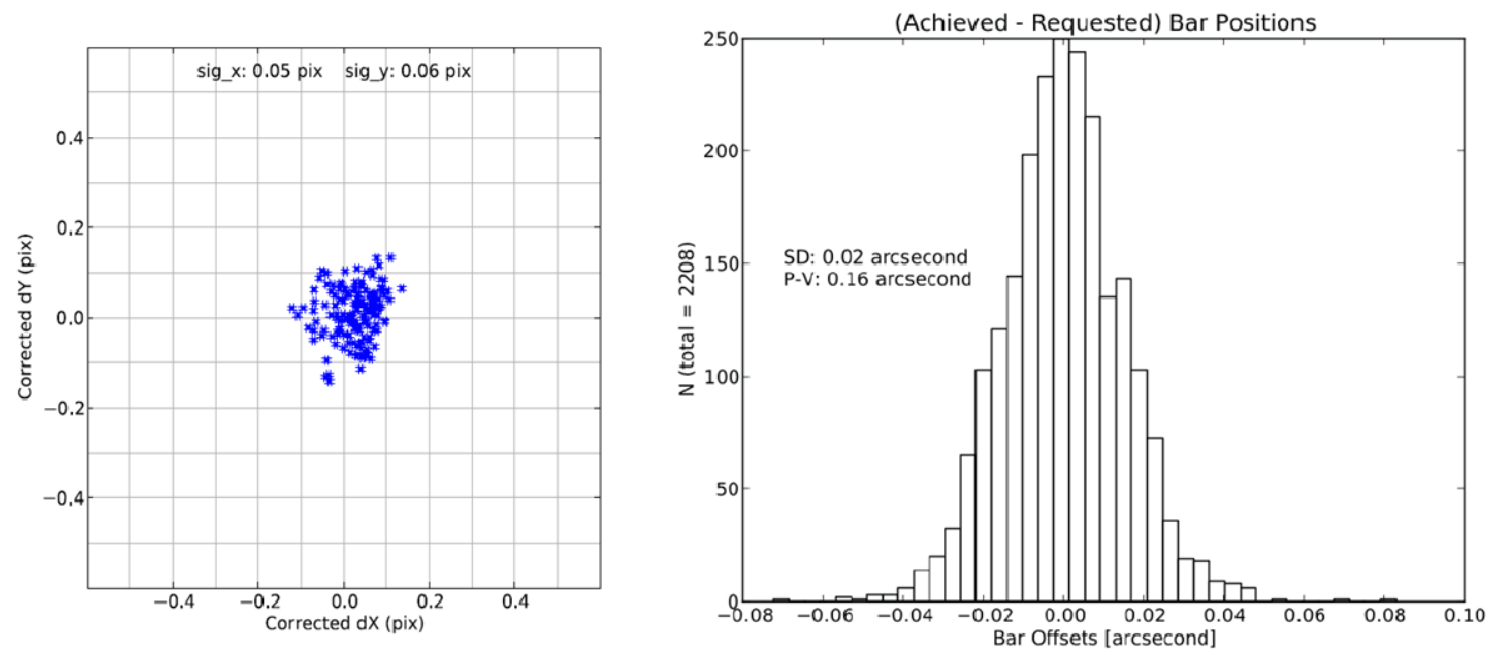

Figure 1. On the left is the typical rms variation in image position through 360 degrees in PA after flexure compensation. On the right is a plot showing the difference between requested and achieved bar positions for the CSU. 
Observations using a pinhole mask in the focal plane and the built-in detector focus mechanism confirm that the optical design delivers $<0.25$ " rms diameter images over the entire field and over the entire wavelength range $(0.97$ to $2.41 \mu \mathrm{m})$ with no refocus needed; pinhole images were $<0.18$ " in some cases. For spectroscopy, the ensquared energy in a $2 \times 2$ pixel box is $80-90 \%$. Throughput estimates, based on as-built parameters for all optical components and the detector, yield $>30 \%$ average over the full bandwidth in each band, and the lowest blaze peak is $>36 \%$. If this is confirmed on the sky then it will exceed requirements. As required, the optical design includes an accessible pupil image and cold stop. The cold stop is implemented with an iris that can be opened to a circle that circumscribes the image of the Keck primary mirror or can be closed down to a hexagon that matches the primary mirror shape. In the latter mode, the mechanism tracks the pupil image. All mechanisms were tested extensively.

Measurements show that light-leakage past the CSU bars, as well as scattered light inside the instrument, are both very low. For example, in 1800 second dark frames, the mean signal is $<0.008 \mathrm{e}^{-} / \mathrm{s} /$ pixel. As specified, the H2-RG detector was successfully implemented using the SIDECAR ASIC (application specific integrated circuit) electronics. With a reverse bias of $300 \mathrm{mV}$ and an amplifier gain giving 2.15 electrons per DN, noise measurements achieve 4.9 electrons rms with 16 Fowler pairs and a floor of 3 electrons rms at 64 Fowler pairs in the lab.

Internally, the optical bench is held at $120 \mathrm{~K} \pm 0.1 \mathrm{~K}$ and the detector is stabilized at $77 \pm 0.01 \mathrm{~K}$. Using three closed cycle refrigerators (CCRs), the instrument cools down passively from ambient in about $\sim 7$ days (Mauna Kea) and thus keeps the average cooling rate for the optics at about $1 \mathrm{~K} /$ hour. The successful double window design allows radiated heat to project outwards onto the large vacuum window to prevent condensation while keeping the inner window cold in order to reduce the radiation load on the slit bars.

Finally, MOSFIRE's software provides a full graphical interface to the user. There are easy means for configuring the CSU and taking exposures. FITS files contain extensions with detailed information on everything in the instrument, including the positions of the masking bars. Slit masks can be created off-line from a prioritized list of targets using the MOSFIRE mask generating software. More details of the as-built performance are provided in the next section.

\section{INTEGRATION AND TESTING}

MOSFIRE's integration and test phase encountered and resolved a number of challenges. For example, shortly after first light in the lab (cool down 5) it was discovered that there were several sources of light leaks inside the dewar due to missing or poorly implemented baffles. A thermal short was encountered when using the detector focus mechanism over its full range, thus requiring the detector head to be dismantled and re-machined. Higher-than-expected friction between the iris blades in the pupil mechanism prevented the iris from closing all the way down to the proper hexagonal shape after initial installation. The shape and support of the blades had to be changed. Most concerning of all was that on each cool down a few CSU bars failed to move correctly or got stuck in the beam. The problem was traced to magnetic debris from cracks in the permanent magnets. The CSU had to be removed for clean up. Fortuitously, this allowed us to install the pinhole mask for optical testing. Finally, we also discovered that the Cassegrain rotator module and velocity servo system was not operating correctly. When reworking part of the cable wrap system did not solve this problem, the real source of friction was traced to the main bearing which had to be replaced. Prior to shipping, all these problems were resolved and the most critical performance characteristics were demonstrated in the lab environment.

\section{Field of View}

The angular size of the sky area imaged onto the MOSFIRE detector is limited by the size of the detector (2040 pixels $\mathrm{x}$ $0.1798 " /$ pixel=366.79" square, projected onto the sky), by the CSU baffle close to the telescope focus, and by a circular baffle in front of the field lens just after the CSU, which has a measured angular diameter projected onto the sky of 414" $\left(6.9^{\prime}\right)$ and corresponds closely to the unvignetted collimator field of view. Thus, the geometry of the MOSFIRE field is a $6.113^{\prime}$ square intersected with a $6.9^{\prime}$ diameter circle.

\section{Imaging Plate Scale}

MOSFIRE's optical system was designed to re-image the Keck Cassegrain $\mathrm{f} / 13.66$ focal plane (nominal scale is 0.72516 $\mathrm{mm} / ")$ onto the H2RG detector $(17.984 \mu \mathrm{m}$ pitch at $77 \mathrm{~K})$ with a plate scale of 0.18 "/pixel, which is a focal reduction of 7.25 times. Assuming that the Keck plate scale is exactly correct the measured pixel size in imaging mode at the detector maps to 0.1798 " on the sky. This mapping was determined during cool down 8 by imaging a mask containing a grid of 
precisely-placed $100 \mu \mathrm{m}$ diameter pinholes (installed during cool down 8 in place of the CSU) through the MOSFIRE optics, and then solving for the geometric transformation from the telescope/CSU focal surface onto the detector. These measurements also allowed us to use MOSFIRE images to calibrate the CSU bar positions. A polynomial mapping solution had a rms of 0.05 pixels in going from CSU to detector, and $0.007 \mathrm{~mm}$ in mapping pixels to $\mathrm{mm}$ in the telescope focal plane. The closest linear mapping solution (i.e., including only a scale factor and rotation) has $\mathrm{rms}=0.18$ pixels (or $0.03 "$ ), or distortion of only $\sim 0.2 \%$. Thus, as predicted, the as-built optics produced nearly undistorted images of the Keck focal surface at the detector. Because the Keck f/ 13.66 focal surface plate scale is effectively linear (with $0.02 \%$ distortion) over the MOSFIRE science field, it should be straightforward to go from detector to CSU to sky coordinate systems using the mapping completed in the lab. This also means that it should be possible to embed accurate world coordinate systems into image headers for direct imaging programs, greatly streamlining those programs requiring mosaics and/or dither patterns, thus enabling nearly-real-time reduction of final, stacked and astrometrically correct images. Happily, these predictions were confirmed at the telescope.

\section{Spectral Resolution}

As built, MOSFIRE meets its spectral resolution specification, achieving $\mathrm{R}=3,380,3,310,3,660$, and 3,620 in $\mathrm{Y}, \mathrm{J}, \mathrm{H}$, and $\mathrm{K}$ bands, respectively for an entrance slit width equivalent to 0.7 " on the sky. These measurements are averaged over the full 3.0' $\mathrm{x}$ 6.1' spectroscopic field, using a single setting of the detector focus which produces direct and spectroscopic image quality within $5 \%$ of their values at optimal wavelength dependent focus settings. For narrower slits, MOSFIRE is capable of still higher spectral resolution: a slit width projecting to 2 detector pixels (0.48") results in measured spectral resolution $\mathrm{R}=4,960,4,930,5,340$, and 5,280 for $\mathrm{Y}, \mathrm{J}, \mathrm{H}$, and $\mathrm{K}$ respectively. Because of the relatively steep angles of incidence for the MOSFIRE spectroscopic modes there is significant compression in the spectral dimension of the dispersed images of slits as recorded by the detector. The (average) anamorphic factors are 1.357 for HK mode, and 1.335 for YJ, so that in spectroscopic mode, one pixel in the dispersion direction maps to $0.1798 * 1.335$ $=0.240 \operatorname{arcsec}$ for YJ and $0.1798 * 1.357=0.244 "$ for HK. Thus, a slit corresponding to $0.6 "$ on the sky produces $0.6 / 0.240(0.244)=2.50(2.46)$ pixel slit image at the detector, which agrees well with measured line widths. The nominal 0.7 " slit would correspond to FWHM $=2.92$ pixels for YJ spectroscopic mode and FWHM $=2.87$ pixels for HK spectroscopic mode in the spectral direction.

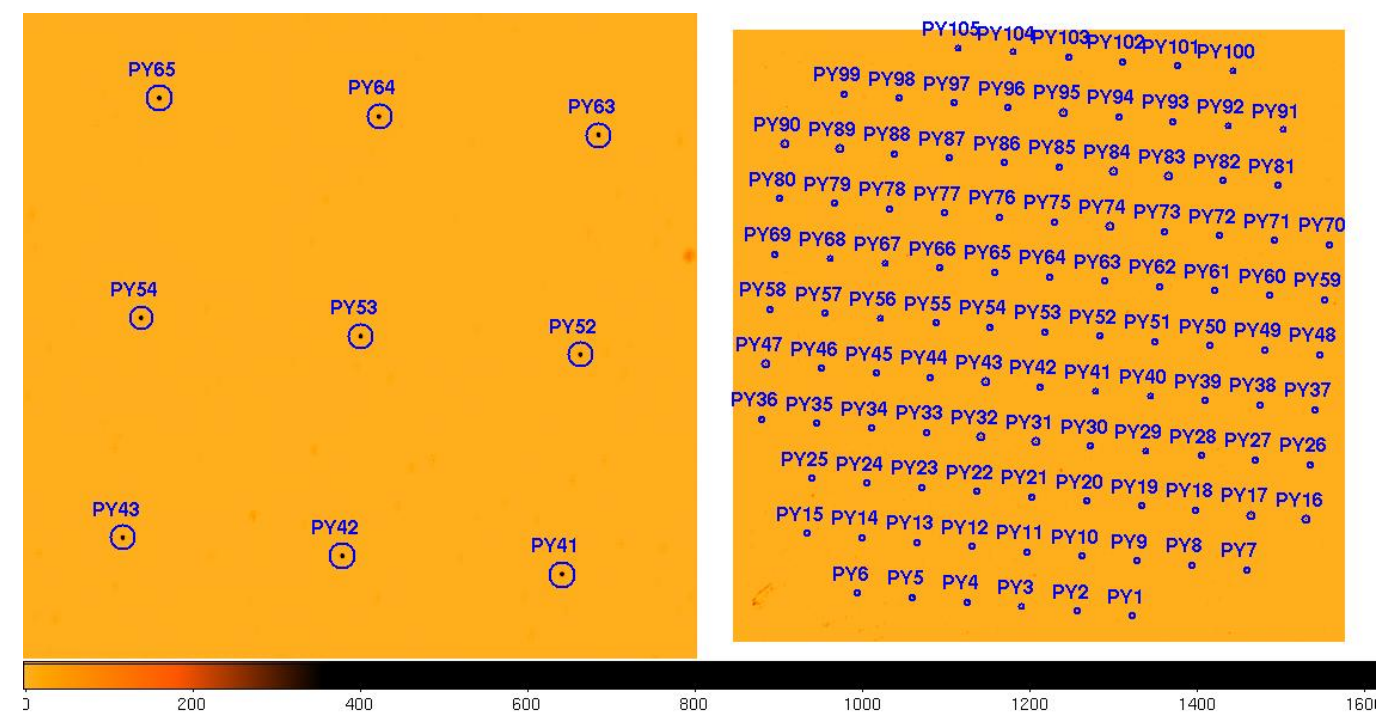

Figure 2: MOSFIRE pinhole mask images. Left: close up view. Right: entire pattern. The pinholes are not resolved.

\section{Image Quality}

After establishing the best detector focus position, pinhole mask images and spectra proved suitable to evaluate the image quality of the as-built system. MOSFIRE's image quality is so good that it is quite difficult to measure the rms image diameter given our pixel sampling at the detector. Measurement of slightly dithered pinhole images allowed us to 
estimate that the rms image diameter produced by a 100 micron pinhole (projecting to $100 / 7.2515=13.8 \mu \mathrm{m}$ or 0.139 ") in imaging mode is $<0.18$ " (averaged over all bands, with no refocus). Example pinhole images are shown in Figure 2. Figure 3 shows the dispersed spectra of the pinholes in the $\mathrm{K}$ band continuum. In spectroscopic mode, the figure of merit is the fraction of light from an unresolved spectral line falling within a $2 \times 2$ pixel box, for which the requirement was greater than $80 \%$ ensquared energy. The as-built system was evaluated using dispersed spectra of arc lamps taken through the pinhole mask. When averaged over the full field, values close to $90 \%$ were achieved.

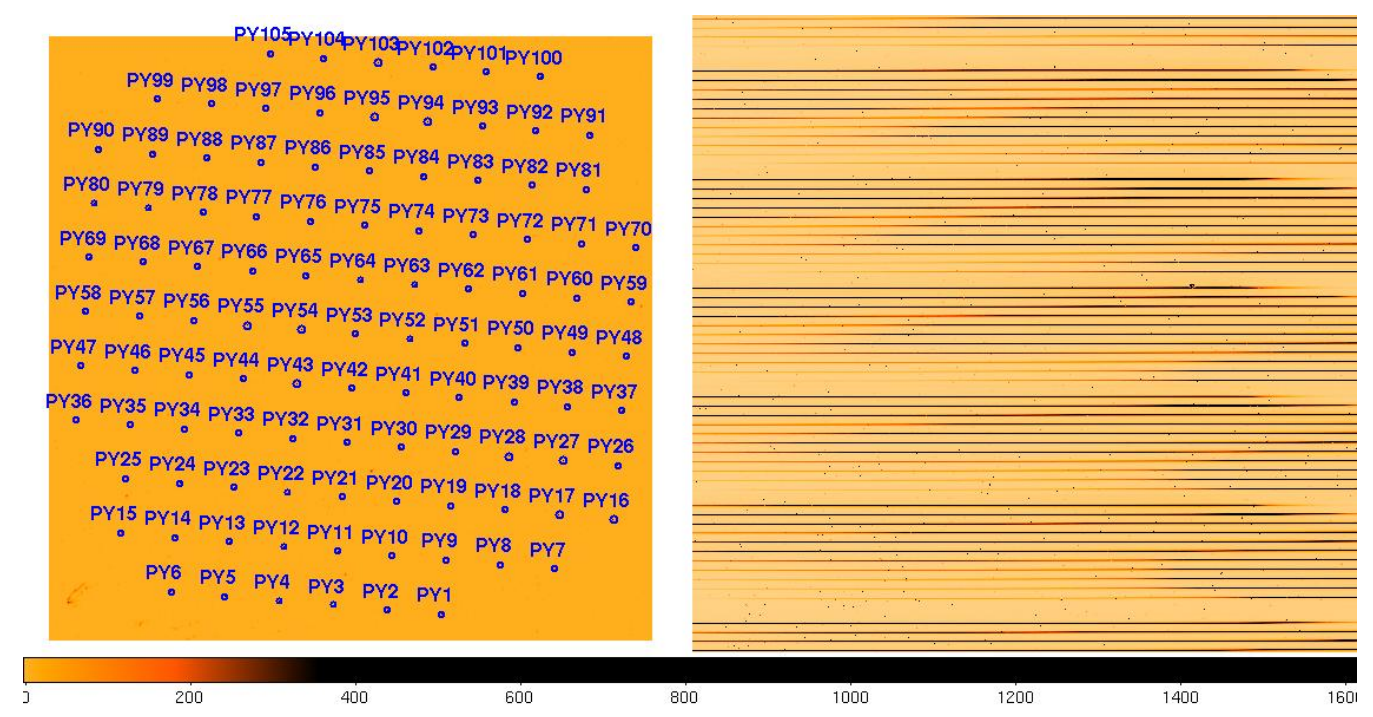

Figure 3. The left side of the figure shows an image of the MOSFIRE pinhole mask with identifying information for each pinhole. The right image shows K band continuum spectra. The spectra are aligned to the detector rows within $0.08^{\circ}$.

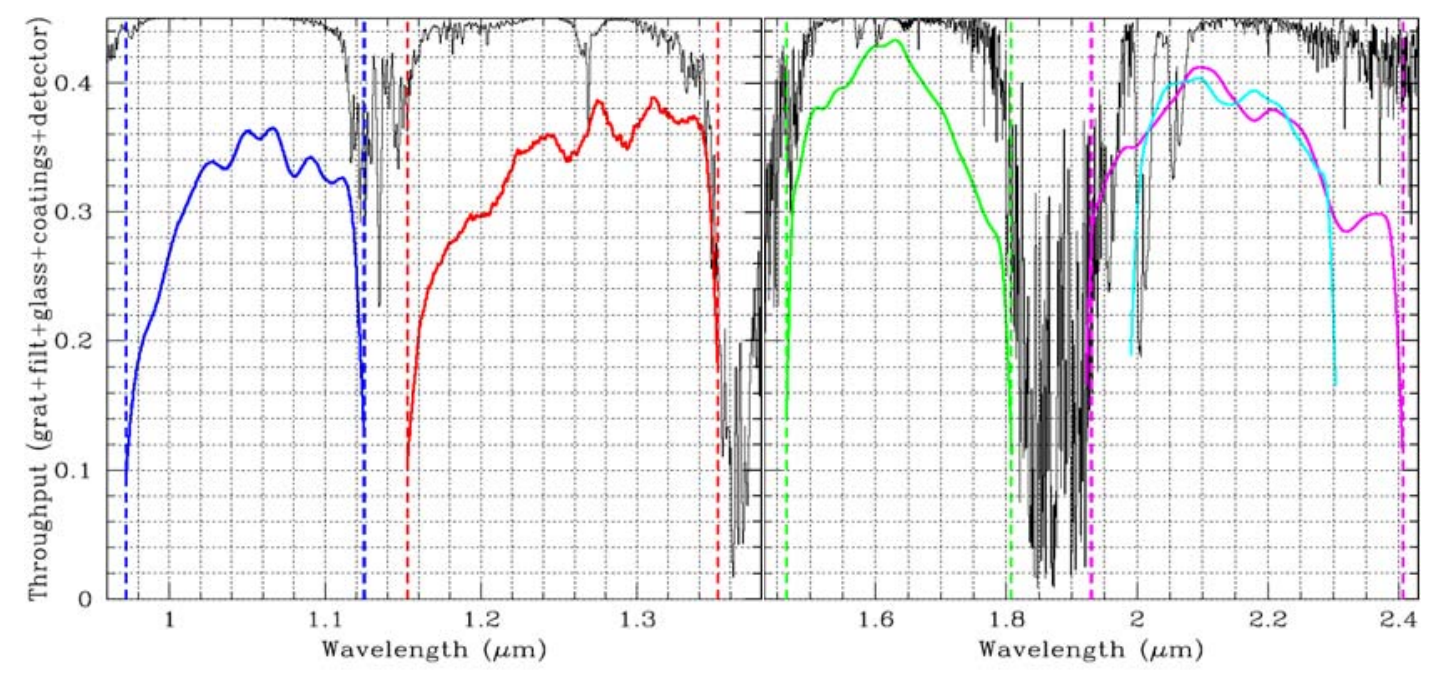

Figure 4. Predicted throughput for each band based on as-built information for the MOSFIRE optics and detector.

\section{Throughput}

MOSFIRE's requirement on throughput from slit to detector is $>30 \%$ on blaze in each band (orders $6,5,4$, and 3 ). We did not have the ability to measure the throughput in the lab, but the as-built data provided by vendors were available for the optical coatings, detector QE, grating diffraction efficiency, and filter transmission. Combining all estimates, the predicted spectroscopic throughput as a function of wavelength in each band is shown in Figure 4 along with the atmospheric transmission (suitably normalized) as a guide to the location of the passbands. In spectroscopic mode, the 
wavelength averaged optical throughput across each band exceeds $30 \%$, with peaks between $36 \%$ and $43 \%$, which easily meets the requirements. Again, we will see that these results were confirmed at the telescope.

Images used to evaluate the CSU's light blocking were obtained with the instrument at the horizon (bars horizontal), in imaging mode, with $\mathrm{Y}, \mathrm{J}, \mathrm{H}$, and $\mathrm{K}$ filters. Very short integrations $(0.25 \mathrm{~s})$ using sub-array readout with illumination from the $\mathrm{Ne}$ arc lamps were made, followed by longer $(120 \mathrm{~s})$ integrations with the even numbered masking bars fully across the field of view. Note that this orientation provides a "worst case" for masking bar "sag", so that the measured level of light leakage should be an upper limit. A portion of the image taken in the $\mathrm{H}$ band is shown in Figure 5 with a negative color mapping, that is, the leakage appears black.

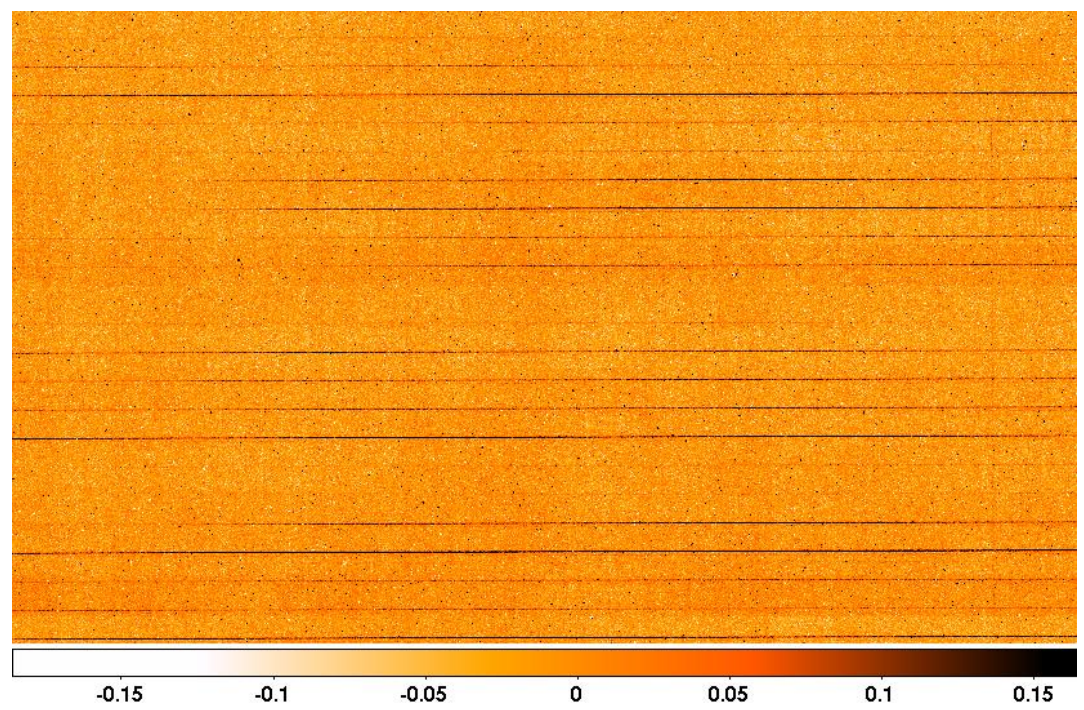

Figure 5: CSU light leak test image; H band, displayed with inverted color scale. Dark horizontal lines are leaks between bars, with peak levels corresponding to $1.4 \times 10^{-5}$ of the baseline signal. Otherwise there is no significant signal measured.

An extensive ghost analysis of the optical design concluded that ghosts should be negligible in imaging mode, and none have been detected. There are, however, features related to grating ghosts in spectroscopic mode. These ghost images may be in-focus images of dispersed spectra reflected from the surface of the detector, re-collimated by the camera, and then reflected back in the $0^{\text {th }}$ order of the grating to be re-focused on the detector.

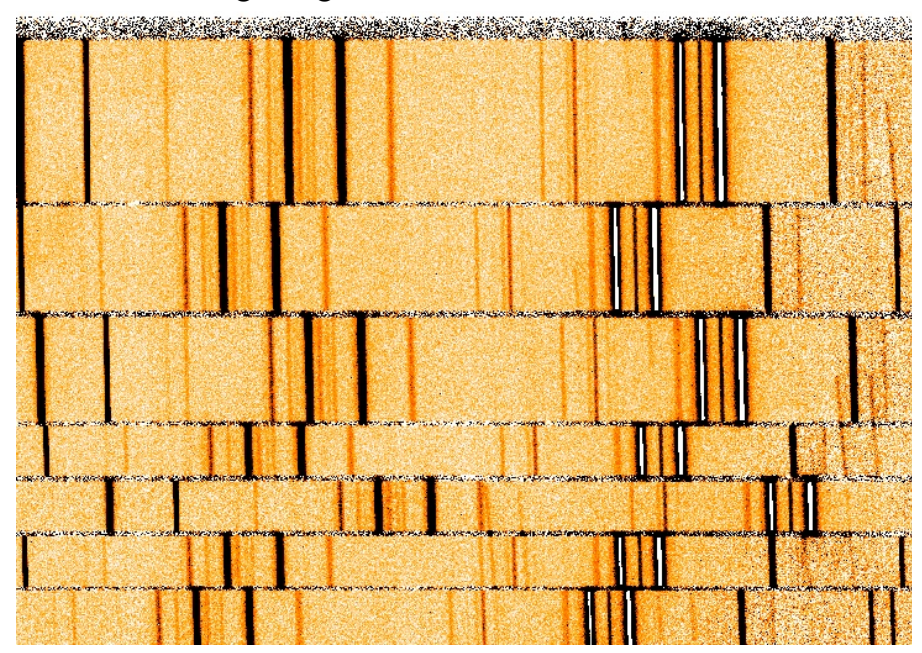

Figure 6: Small portion of a Y-band arc spectrum. Faint ghost features that are $0^{\text {th }}$ order back-reflections of the bright saturated pair of lines on the right side of the image can be recognized by their tilts compared to the primary image. 
These ghosts were anticipated and are an unavoidable consequence of the geometry, which has the grating normal only a few degrees from the optical axis, meaning that some spectra, from some slits, will appear as focused ghosts on the detector. A careful analysis of arc lamp spectra taken during lab integration led to measurements of the ghost intensity that is between $1.6 \times 10^{-3}$ and $0.5 \times 10^{-3}$ of the primary signal (in practice, they can only be detected for lines that are strongly saturated in the minimum exposure time). Figure 6 shows an example. We do not expect these ghosts to have a significant effect on science with MOSFIRE.

\section{Filters}

MOSFIRE has four broadband order-sorting filters, $\mathrm{Y}, \mathrm{J}, \mathrm{H}$ and $\mathrm{K}$, with the $\mathrm{Y}, \mathrm{J}$, and $\mathrm{H}$ filters doubling as photometric filters for imaging. A Ks filter is also provided for imaging or spectroscopy. During the development phase, Alice Shapley (UCLA) purchased four additional intermediate-band filters, called J2, J3, H1, and H2. These filters, envisioned primarily as imaging filters, roughly split the $\mathrm{J}$ and $\mathrm{H}$ bands in half, and are part of an intermediate band filter system described by van Dokkum et al. (2009) The Y band filter already supplied with MOSFIRE is essentially identical to the "J1" filter of that intermediate band system. An additional narrow band filter $(0.2 \%$ at $1.0625 \mu \mathrm{m})$ provided by Elizabeth Barton (UCI) was added just before delivery. A "dark" configuration is formed using two disjoint filters in FW1 and FW2 simultaneously. All filters were manufactured by Barr Associates according to specification. All MOSFIRE filters meet our requirements for throughput, bandpass edge slope, flatness, and ripple. All filters achieve uniformly excellent image quality. Digital versions of the filter passbands are available on the Keck instrument pages for MOSFIRE.

\section{Grating}

As specified in the requirements, the MOSFIRE spectrometer uses a reflection grating that is interchangeable with a plane mirror for imaging and two grating angle stop positions are provided (one for the $\mathrm{Y}$ and $\mathrm{J}$ bands, one for the $\mathrm{H}$ and $\mathrm{K}$ bands) to center the relevant spectral order within each near-IR atmospheric band. The grating was custom ruled specifically for the MOSFIRE application in orders 3, 4, 5, and 6 with a $40^{\circ}$ included angle and angle of incidence (AOI) of $42.6^{\circ}$ ( $\mathrm{H}$ and $\mathrm{K}$ ) and $41.5^{\circ}$ (Y and $\mathrm{J}$ ). The grating has 110.5 lines $/ \mathrm{mm}$ with a blaze angle of $21.93^{\circ}$ (first order blaze of $6.35 \mu \mathrm{m})$, and was replicated onto a Clearceram ${ }^{\circledR}-\mathrm{Z}$ (OHARA) substrate, with a gold-coated ruled surface. The master and replica were manufactured by Newport Optical (Richardson Gratings) and all specifications were met. Table 1 lists the achieved central wavelengths and observed wavelength ranges for slits centered in $X$, and at $+/-1.5$ ' that represent the typical region that will be used for slits under normal circumstances.

Table 1: MOSFIRE spectral coverage as-built

\begin{tabular}{|c|c|c|c|c|c|c|}
\hline Band & $\begin{array}{c}\text { Dispersion } \\
\text { (A/pixel) }\end{array}$ & $\begin{array}{l}\lambda \text { center, } \\
A \text { (note } 1)\end{array}$ & $\begin{array}{c}\text { Range at center, } \\
A\end{array}$ & $\begin{array}{l}\text { Range at }-1.5^{\prime} \\
\text { (see note } 2 \text { ) }\end{array}$ & $\begin{array}{l}\text { Range at }+1.5^{\prime} \\
\text { (see note } 2)\end{array}$ & $\begin{array}{l}\text { Shift, A/mm } \\
\text { (see note 3) }\end{array}$ \\
\hline $\mathrm{K}$ & 2.1691 & 21760 & 19540 to 23970 & $\begin{array}{c}20340 \text { to } 24060 \\
(82 \%)\end{array}$ & $\begin{array}{c}19210 \text { to } 23170 \\
(82 \%)\end{array}$ & 12.209 \\
\hline $\mathrm{H}$ & 1.6269 & 16321 & 14680 to 18040 & $\begin{array}{c}15280 \text { to } 18090 \\
(82 \%)\end{array}$ & $\begin{array}{c}14640 \text { to } 17440 \\
(82 \%)\end{array}$ & 9.157 \\
\hline $\mathrm{J}$ & 1.3028 & 12450 & 11530 to 13520 & $\begin{array}{c}11650 \text { to } 13520 \\
(94 \%)\end{array}$ & $\begin{array}{c}11530 \text { to } 13520 \\
(100 \%)\end{array}$ & 7.460 \\
\hline Y & 1.0855 & 10373 & 9716 to 11250 & $\begin{array}{c}9716 \text { to } 11070 \\
(88 \%)\end{array}$ & $\begin{array}{c}9716 \text { to } 11250 \\
(100 \%)\end{array}$ & 6.216 \\
\hline & $\begin{array}{cl}\text { tes: } & \\
\text { 1. } & \text { Centra } \\
\text { 2. } & \text { Wave } \\
& \text { wavel } \\
\text { 3. } & \text { Shift }\end{array}$ & length & $\begin{array}{l}\text { lit at the center o } \\
\text { placed at } X= \pm \\
\text { al slits covered } \\
\text { ngth per delta }(X\end{array}$ & $\begin{array}{l}\text { field. } \\
\text { rom field center. } \\
\text { spectra from slit } \\
\mathrm{nm} \text { at the CSU, }\end{array}$ & $\begin{array}{l}\text { umber in parenth } \\
\text { e extrema. } \\
\text { stroms. }\end{array}$ & is the fraction of the \\
\hline
\end{tabular}

As shown in Table 1, moving a slit in the CSU (i.e. the telescope) focal plane will cause a shift in the spectral format as seen by the detector. The second column is the linearly approximated dispersion in $\AA$ /pixel, the third column is the wavelength recorded at the center of the detector $(X$ pixel $=1024)$ and the next three columns show the full wavelength range that will be recorded at $X=0,-1.5^{\prime}$, and $+1.5^{\prime}(0,-65.2635 \mathrm{~mm},+65.2635 \mathrm{~mm}$, respectively). The prediction for the mapping of CSU slit position (in $\mathrm{mm}$ ) to wavelength coverage in a given band has been incorporated into the MOSFIRE 
mask design tool MASCGEN. This allows the observer to preview the spectral layout that would result from a spectroscopic observation in the selected band $(\mathrm{Y}, \mathrm{J}, \mathrm{H}, \mathrm{K}$, or $\mathrm{Ks})$ band using the currently loaded mask configuration.

\section{Focus Control}

Although MOSFIRE was designed with a remotely controlled detector focus mechanism that was operated successfully during cool downs 5,7, and 8, a detailed analysis of spectral line widths (and pinhole mask images for imaging mode) distributed over the nominal 3.0' $\mathrm{x}$ 6.1' spectroscopic field revealed that the instrument is so close to being parfocal in all bands and all modes that we have chosen to fix the focus stage at a single value of -200 steps from nominal (each step corresponds to $\sim 0.07 \mu \mathrm{m}$ in the axial direction). Table 2 shows for each band and for the average of all bands the dispersion of best focus values over field in steps, the average line width at best focus, and the average line width for a 0.60 " equivalent entrance slit at the nominal focus position of -200 steps, using slits distributed over the nominal spectroscopic field of view, and over the full wavelength range covered by each slit. The dispersion in best focus values shows that the differences from band to band are at most only marginally significant. By using a fixed focus setting, the average line widths remain very close to their bandpass optimized values (column 4) and we mitigate the risk of a mechanical failure in the focus stage, which would be a possible single-point failure mode.

Table 2: MOSFIRE best overall focus

\begin{tabular}{c|c|c|c}
\hline Band & $\begin{array}{c}\text { Best focus position } \\
\text { in steps }\end{array}$ & $\begin{array}{c}\text { Average line FWHM } \\
\text { at best focus (pixels) }\end{array}$ & $\begin{array}{c}\text { FWHM at -200 steps } \\
\text { (pixels) }\end{array}$ \\
\hline \hline $\mathrm{K}$ & $-300 \pm 80$ & $2.25 \pm 0.12$ & 2.42 \\
\hline $\mathrm{H}$ & $-210 \pm 80$ & $2.38 \pm 0.12$ & 2.39 \\
\hline $\mathrm{J}$ & $-10 \pm 70$ & $2.40 \pm 0.21$ & 2.51 \\
\hline $\mathrm{Y}$ & $-410 \pm 80$ & $2.25 \pm 0.12$ & 2.46 \\
\hline average & $-230 \pm 170$ & $2.35 \pm 0.26$ & $2.45 \pm 0.18$ \\
\hline
\end{tabular}

\section{Guider}

As specified in the requirements MOSFIRE is provided with an optical guider with remote focus control. The MOSFIRE guider provides an offset FOV adjacent to the science FOV. The MOSFIRE science FOV is 6.9' in diameter and is centered on the telescope optical axis. The guider FOV is $2.8^{\prime} \times 2.8^{\prime}$ and is offset 6.7' from the center of the MOSFIRE science FOV. MOSFIRE's guider is controlled by the Observatory's standard MAGIQ software. The camera is equipped with an E2V CCD47-20BT detector. This detector is mounted on MOSFIRE at the rear of the guider optics assembly. A focus mechanism controlled by a Galil DMC-4010 motion controller is provided. MOSFIRE's guider has a single fixed passband with a long pass RG780 filter. To calculate the sensitivity we define the passband with a cut-on wavelength or half-power point of $780 \mathrm{~nm}$ and a cut-off wavelength of $1000 \mathrm{~nm}$, the wavelength where the CCD response has dropped to $\sim 15 \%$. Assuming an average reflectivity for the two mirror surfaces of the Keck telescope we calculate an average throughput over the passband of 61\%. Using the published QE curves for the e2v CCD47-20 back thinned AIMO CCD with a mid-band coating and the performance of the as-built MOSFIRE guide camera head with a gain of setting of three, we find that a magnitude of 20 in the defined passband results in an SNR of 10 for a one second exposure.

\section{Mechanical}

MOSFIRE is heavier than specified by $150 \mathrm{~kg}$, but this additional weight can be accepted by the Observatory. The excess can be balanced out by adding weight to the top end of the telescope, which in turn will require adding weight to LRIS to restore the balance in order to avoid top end weight changes. Physical dimensions, flexure, thermal properties and alignment are all within requirements.

The maximum filter wheel mechanism cycle time is approximately double the values specified in the requirements, but since multiple mechanisms are usually moved simultaneously, and overhead associated with re-configurations will generally be dominated by the time taken to reposition the CSU bars, somewhat slower filter moves will not be significant. The rotation range of the rotator is limited to slightly less than the $530^{\circ}$ specified, but the as-built range still allows sufficient travel to meet observing requirements. We did not provide a means to allow removal of the getter without disturbing the instrument vacuum and its construction does not allow the canister to be baked along with the contents. However, we do not believe that getter service will be a frequent event, and normally will occur only when the instrument is opened for servicing, making the more limited access acceptable. 


\section{Flexure}

The requirement for flexure correction is that over the course of any 2-hour track on a science target, the image motion at the detector due to flexure must be smaller than 0.3 pixels. At the latitude of the Observatory the expected change in rotator angle in a 2 hour period is a maximum of $\sim 60^{\circ}$, with a change in elevation $<30^{\circ}$. The goal requirement is for image motion to be less than 0.1 pixels at the detector for all rotator angles and all telescope elevation angles. It is also highly desirable that the positions of slits at the detector remain stable to sub-pixel levels to allow flat-field calibrations to be obtained for a given configuration during the afternoon or early morning. As built, the instrument flexure in the dispersion direction (instrument $\mathrm{X}$ ) is close to FEA predictions ( \pm 1.2 pixels), but the as-built flexure of the instrument in the Y direction (spatial, along slits in spectroscopic mode) is significantly larger than predicted. Using extensive tests in the lab, the behavior of the flexure was successfully modeled. Once the model parameters are measured and verified at several elevation angles, only the elevation angle and the rotator PA are needed to predict the flexure to significantly less than 0.1 pixels at the detector. The models are slightly different for each mode (imaging mirror, YJ spectroscopy, HK spectroscopy) but the appropriate model is automatically selected when the user switches between modes.

We have verified that flexure correction allows for maintaining images at a fixed detector position for all elevation angles $>22^{\circ}$ (airmass $<2.67$ ) for all rotator angles. Part of the elevation $=0^{\circ}$ ellipse lies outside the FCS range, leaving 2 to 3 pixels of uncorrected Y-direction flexure at particular rotator position angles. However, by having a "home" position angle when the telescope is pointed to the horizon stow position (by design this is a PA at which the CSU masking bars are horizontal), then with the FCS enabled calibrations may be obtained even in the horizon stow position with full flexure compensation.

\section{Configurable Slit Unit}

The CSU was first installed and operated in MOSFIRE during cool down 3 in November and December 2009 and since that time over 1000 mask configurations have been made. Based on difficulties encountered with reliable operation during cool downs 5 and 7, evidence was found that the magnets used to actuate the CSU's slit bar ratchet clutches and brakes were cracking, creating magnet debris. The magnets were subjected to excessive stress due to the difference in rate of thermal expansion and contraction between the magnet and the aluminum frame of the brake or clutch in which the magnet was mounted. The result was one complete failure of a ratchet clutch (bar 75) when the solenoid coil that engaged the clutch was shorted by a piece of sharp debris from an adjacent magnet, and intermittent operation of other bars due to mechanical interference between adjacent clutch or brake assemblies caused by the magnetic debris.

After CSEM preformed an investigation we held a review in October 2010 where we asked an external committee to review the status of the CSU and the problems with the cracking magnets and recommend a course of action. The result was a recommendation to disassemble the CSU and attempt to remove the debris and correct any damage that may have resulted. This work was performed by CSEM during the period of late November 2010 to mid-February 2011.

The repair work by the CSEM engineers required full disassembly of the CSU and therefore ran the risk of losing the precision calibrations (better than $\pm 10 \mu \mathrm{m}$ positioning accuracy) of each bar's motion which CSEM had carefully performed prior to delivery. CSEM used a three-axis CMM and machine vision system to perform the calibration in their clean room facility. No such system was available for use in the on-site repair work at CIT. However, MOSFIRE's imaging system had already been demonstrated to be capable of very precise measurements of the CSU positions in the focal plane. We had also planned to install a pinhole mask at MOSFIRE's focal plane in place of the CSU in order to fully characterize the focal plane distortions, but we had not done so due to the need for more extensive testing of the CSU through cool down 7. We took advantage of the period in which the CSU was being worked on to perform cool down 8 using a precisely-machined mask with $100 \mu \mathrm{m}$ pinholes in place of the CSU. This allowed accurate mapping of the instrument's focal plane, and additional characterization of the instrument's optics and spectral calibration.

Based on images taken through the pinhole mask and recorded on the detector after all adjustments had been made to the detector mount and the positions of the grating and imaging mirror, we obtained a geometric solution that maps the telescope focal surface to precise pixel locations at the detector. The transformation allowed measurement to $\sim 6 \mu \mathrm{m}$ accuracy of locations on that surface using measured image locations on the detector. During cool down 9, for which the CSU was re-installed, extensive measurements of bar positions versus requested CSU positions were made. The typical bar accuracy over the nominal 3.0' $\mathrm{x} 6.12^{\prime}$ spectroscopic field is $\sim 6 \mu \mathrm{m}$ in the CSU plane, mapping to $<0.02^{\prime \prime}$ arc seconds on the sky and 0.07 pixels at the detector, thus easily exceeding the accuracy requirement and matching the laboratory 
measurements made by CSEM. The new calibration also has the advantage that it is based on measurements made at $120 \mathrm{~K}$, rather than the room temperature measurements used for the initial calibration.

\section{ON-SKY PERFORMANCE}

After ten cool downs had demonstrated that MOSFIRE meets all of its requirements, the instrument was prepared for shipping to Hawaii. Following its arrival on Mauna Kea on February 16, MOSFIRE was carefully examined, then pumped out and cooled down for the eleventh time. After a series tests on the Nasmyth deck and several practice insertions into the Cassegrain focal station, the instrument achieved "first light" during a two-night run beginning on April 4, 2012. Unfortunately, the weather was cloudy on both nights but seeing conditions were good. Figure 7 shows our first light target, the Antennae galaxies (NGC40xx), as well as our first real on-sky mask.
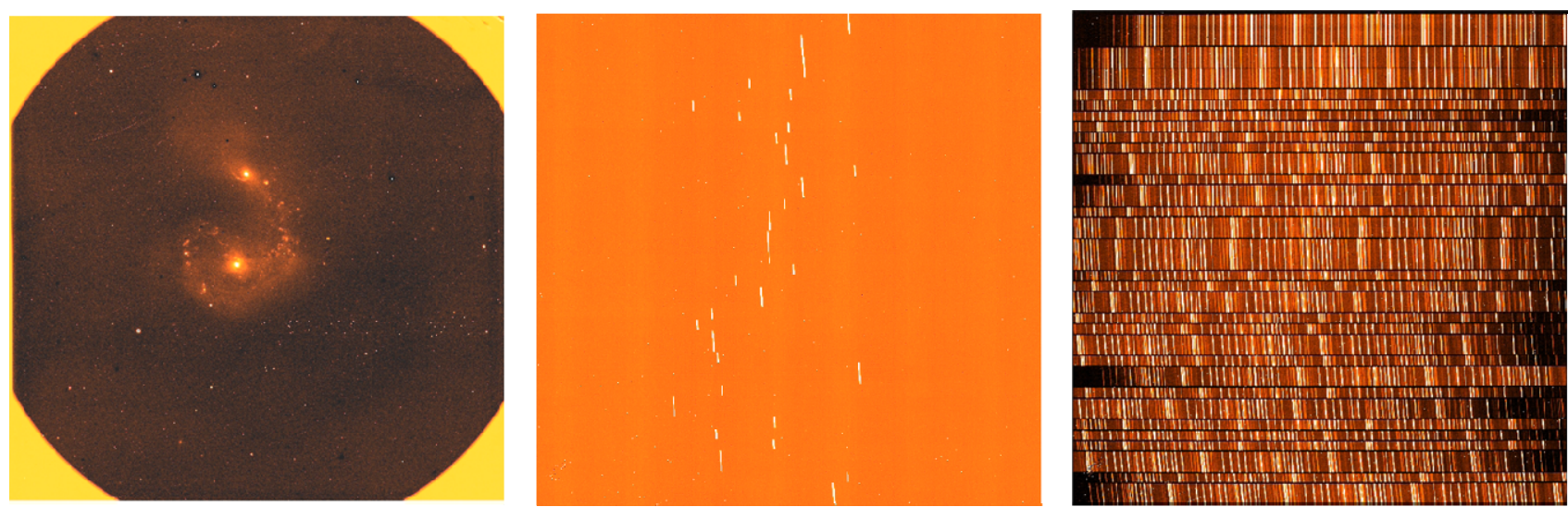

Figure 7. On the left is the layout of the MOSFIRE field on the sky with a 58s J-band image of The Antennae galaxies. The middle image is of a slit mask and the right image is the night sky emission with this mask in H-band.

Despite the cloudy skies we were able to demonstrate outstanding image quality during the first light run and subsequent commissioning nights. Figure 8 shows a typical FWHM value of $0.45^{\prime \prime}$ (at J) for stars in the globular cluster NGC5053. Even better images were measured in the Ks band.

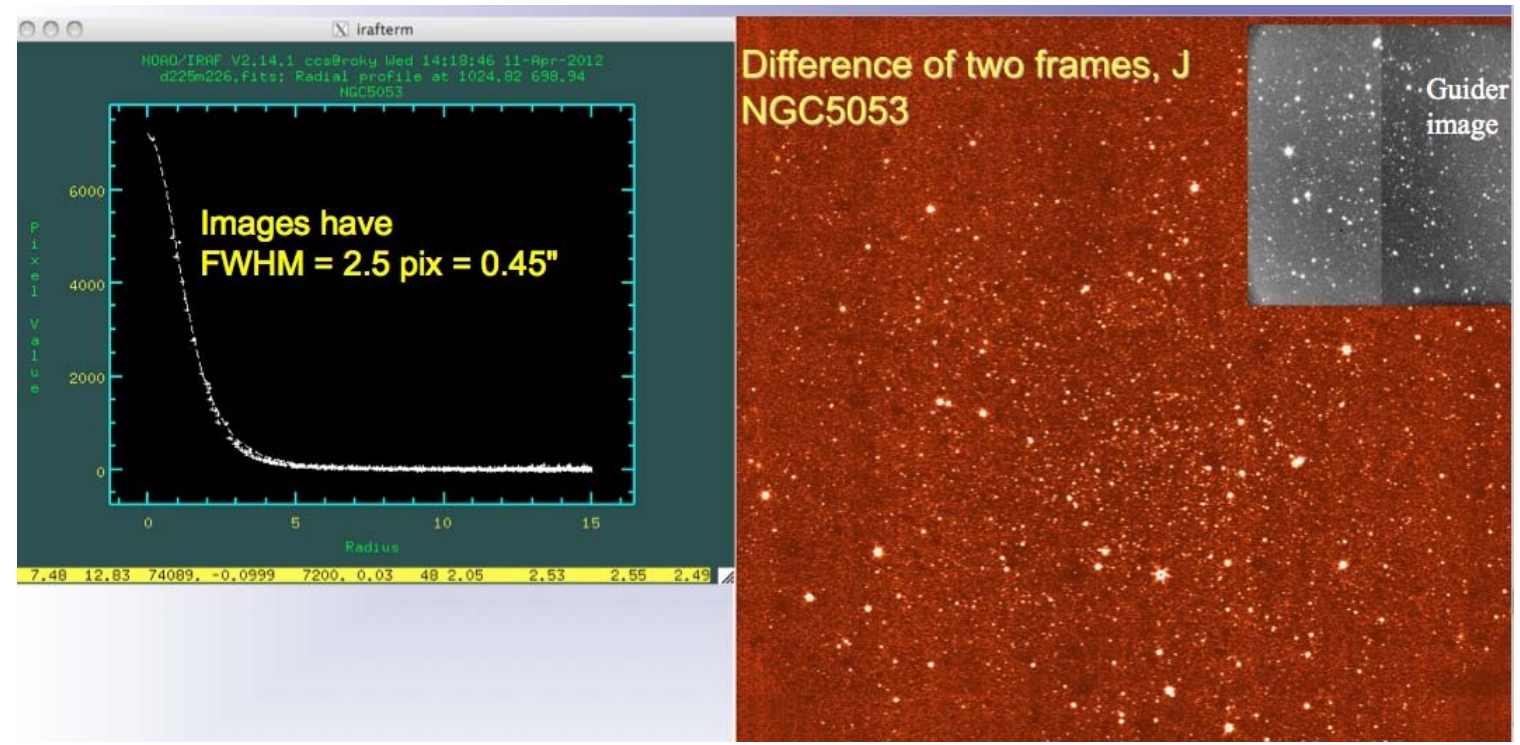

Figure 8. A J-band exposure on the globular cluster NGC5053 gives an average FWHM of 0.45". The best images were recorded in K-band where the FWHM was $<2$ pixels, or $<0.36$ " FWHM. 
The MOSFIRE pixel scale has been measured to be consistent with linear over the entire field of view, with a measured plate scale of $0.1798^{\prime \prime} /$ pixel. All MOSFIRE images are populated with world coordinate system information in their headers that provide immediate access to orientation, handedness, and astrometry. The same globular cluster stars shown in Figure 8 were used to map the distortion and derive an astrometric solution for the CCD guide camera located 6.7' off axis. The guider has up to $10 \%$ distortion which increases with field angle, thus the distortion map will be incorporated into the MAGIQ guider software to allow for the most accurate telescope offsets over the entire $2.9^{\prime}$ x $2.9^{\prime}$ guider field. Once the Slit Alignment Tool has been used to position the telescope correctly and set the instrument PA to place objects in slits, the offset guider does an excellent job of holding objects in 0.7 " slits for many hours.

At pre-ship review we estimated the throughput of MOSFIRE alone, that is, the throughput from slit to detector without including the telescope optics or atmospheric transmission. The predicted average values for the $\mathrm{Y}, \mathrm{J}, \mathrm{H}$ and $\mathrm{K}$ spectroscopy bands were $0.308,0.325,0.361$ and 0.350 respectively. These estimates included glass transmission, grating efficiency, and anti-reflection coating on glass and mirrors as well as the detector quantum efficiency. Figure 9 shows the measured "on-sky" throughput for each of these bands. Clearly the agreement is remarkably good!
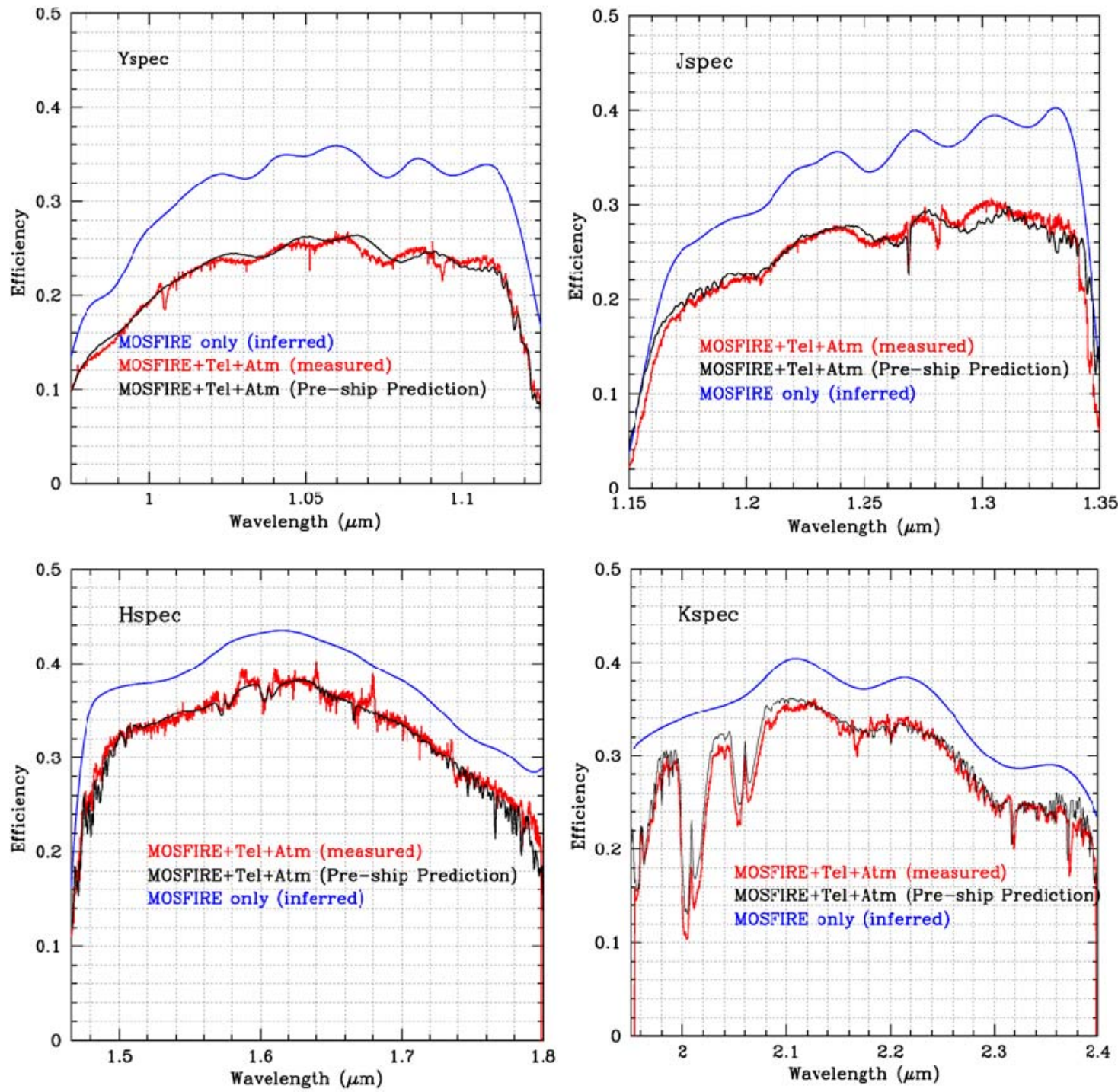

Figure 9. Measured throughput (red) compared to predictions (black) as a function of wavelength for each of the four spectroscopic bands, Y, J, H and K. Blue curves give the inferred throughput of MOSFIRE alone in spectroscopic mode. 
MOSFIRE's flexure compensation system is intended to keep images or spectra fixed with respect to the detector to within $<0.1$ pixels $\left(<0.018^{\prime \prime}\right)$ rms. In fact, we found that data taken using the same slit mask in May and June could be reduced using the same calibration data, indicating that in addition to the FCS image stability, both the CSU and the grating are highly repeatable.

Measurements of the sky background for imaging and spectroscopy have been made. All observations were carried out during full moon, which does have an effect for the shortest wavelengths. Tables 3 and 4 give the sky in magnitudes per square arcsecond in both the Vega and $\mathrm{AB}$ systems for the imaging (Table 3) and spectroscopic (Table 4) modes. The average sky intensity in units of electrons per spatial pixel per second between the $\mathrm{OH}$ lines is also given in column 4 of Table 4; the low count rates reflect one of the major goals in the design of MOSFIRE: to take advantage of the very low terrestrial background for spectral regions away from strong $\mathrm{OH}$ emission lines. Note (for example) that in $\mathrm{H}$ band the background reduction factor is $\sim 2.7$ magnitudes in comparison to the broadband background (and a factor of $\sim 500$ times lower than the brightest $\mathrm{OH}$ line in $\mathrm{H}$ band). All of these numbers are consistent with our expectations and assumptions.

Table 3. Sky brightness in imaging mode (mags/sq. arcsec)

\begin{tabular}{|l|l|l|}
\hline Band & Vega & AB \\
\hline Y & $17.37-17.50$ & $18.02-18.15$ \\
\hline J & $15.49-15.87$ & $16.39-16.77$ \\
\hline H & $13.59-13.89$ & $14.99-15.29$ \\
\hline Ks & $13.68-14.25$ & $15.54-16.11$ \\
\hline
\end{tabular}

Table 4. Sky brightness between $\mathrm{OH}$ lines in spectroscopic mode (mags/sq. arcsec)

\begin{tabular}{|l|l|l|l|}
\hline Band & Vega & AB & e-/s/pixel \\
\hline Y & 18.73 & 19.38 & 0.34 \\
\hline $\mathrm{J}$ & 18.34 & 19.24 & 0.30 \\
\hline $\mathrm{H}$ & 17.32 & 18.72 & 0.57 \\
\hline $\mathrm{K}$ & 16.35 & 18.21 & $0.39(\lambda<2.2 \mu \mathrm{m})$ \\
\hline $\mathrm{K}$ & 14.05 & 15.91 & $8.0(2.35-2.41 \mu \mathrm{m})$ \\
\hline
\end{tabular}

Note: the fourth column is the observed background between $\mathrm{OH}$ lines, per spatial pixel, for a 0.7 " slit.

MOSFIRE's sensitivity for imaging and spectroscopy also depends on the high QE, low noise performance of the detector. Our goal for detector dark current was $<0.01 \mathrm{e}-/ \mathrm{s} /$ pixel, while we expected (with the aide of many multiple non-destructive reads, also known as multiple correlated double sampling [MCDS] or Fowler sampling), that the effective read noise could be reduced to $\sim 3 \mathrm{e}$ - rms. In the lab, long dark exposures measured a value of $<0.008 \mathrm{e}-/ \mathrm{s} / \mathrm{pixel}$ for the average dark current, and a sequence of noise measurements using increasing numbers of non-destructive reads demonstrated that $3.2 \mathrm{e}-\mathrm{rms}$ was achievable with 64 reads, while 16 reads was sufficient to get below 6 e- rms. A single read, also called a correlated double sample (CDS) gave about $17.2 \mathrm{e}-\mathrm{rms}$ in the lab. The observatory environment is electrically noisier. In CDS mode the measured read noise is $23.2 \mathrm{e}-\mathrm{rms}$, but 16,32 and 64 reads essentially yield noise within $\sim 10 \%$ of lab values. This performance ensures that MOSFIRE becomes background-limited (even in the darkest sky regions between $\mathrm{OH}$ lines) in a few minutes for all four spectroscopic bands; dark current is always negligible.

To illustrate the MOSFIRE spectroscopic mode, Figure 10 shows part of a mask used to observe high redshift galaxies in $\mathrm{K}$-band looking for $\mathrm{H}$-alpha emission. After the mask has been aligned, typical observations will involve nodding the telescope in the direction along the slit to place each target at two or more spatial positions. In this case, a "mask nod" pattern of $\pm 1.5^{\prime \prime}$ leads to a 3 " separation between A and B positions, which alternate after each 180 s integration. A crude background subtraction is achieved by stacking all of the exposures taken at the A position, and all those at the B 
position, and simply subtracting one from the other (we refer to this as an "interleaved difference image"). Figure 10 shows the result for such an interleaved difference in which there were five 180s integrations at each position (i.e., a total of 1800 s on source).

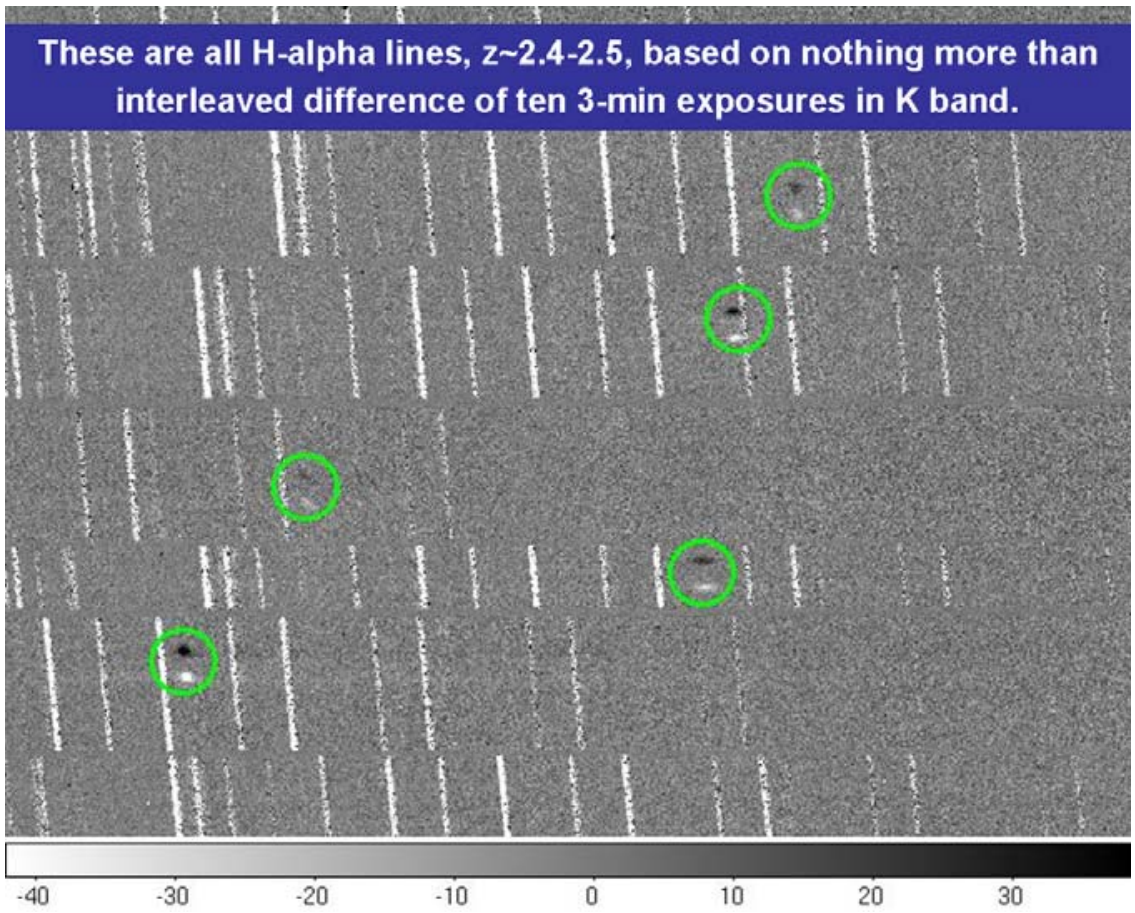

Figure 10. H-alpha lines at $\mathrm{z}=2.4-2.5$ based on 30 minutes of exposure. Nodded pairs produce positive and negative images.

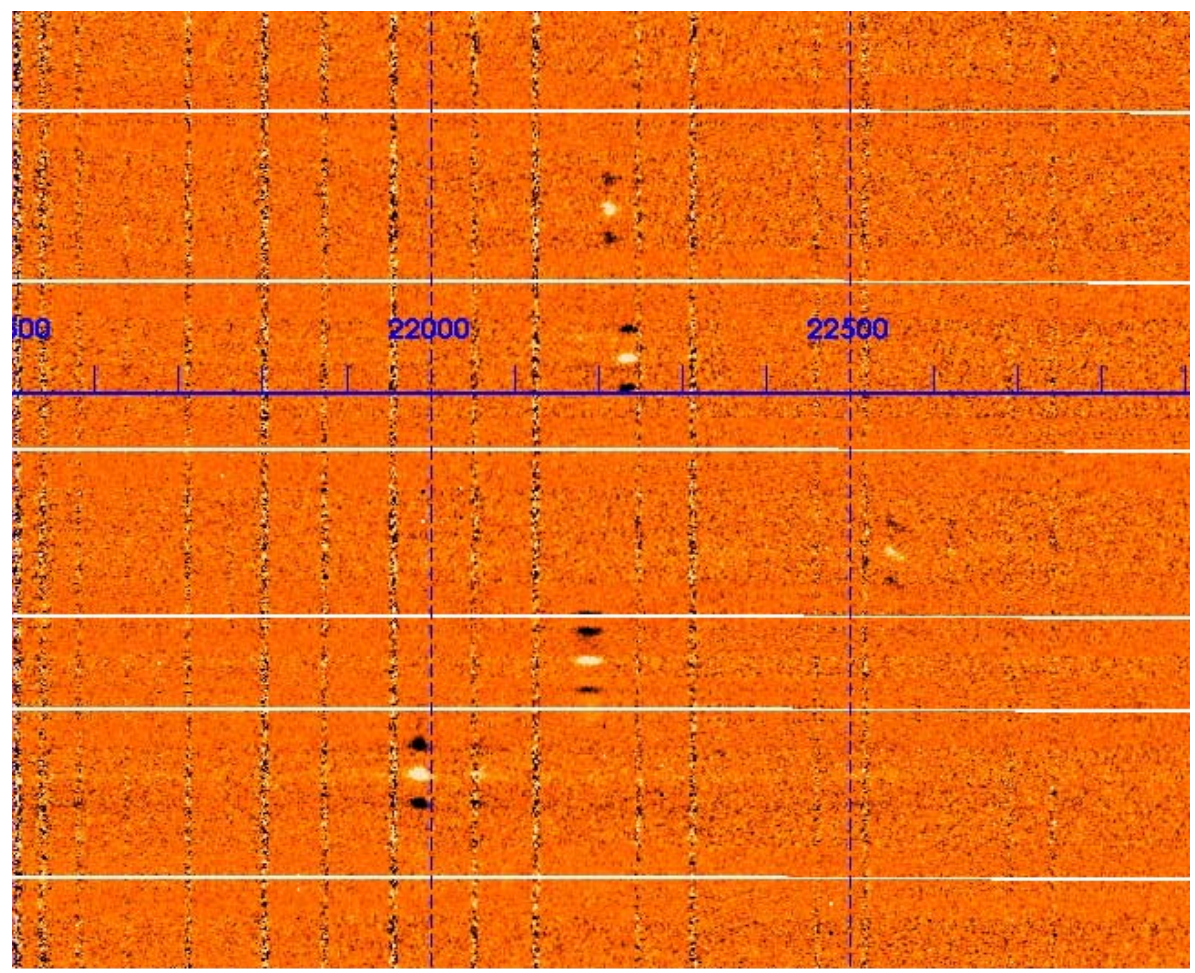

Figure 11. The same mask observations as in Figure 10 but after passing through the Data Reduction Pipeline. Double negative images are due to the fact that the A and B signals have now been shifted and added together. 
The MOSFIRE Data Reduction Pipeline (DRP) has been developed by the MOSFIRE team and has undergone substantial testing during commissioning. It is designed to take a sequence of spectroscopic exposures (which will typically be obtained using two or more nod positions in an alternating pattern) and automatically produce flat-field corrected, background subtracted, wavelength calibrated, and rectified 2D spectra, along with products useful for quantifying the data quality. Figure 11 shows the mask observations from Figure 10 after passing through the DRP.

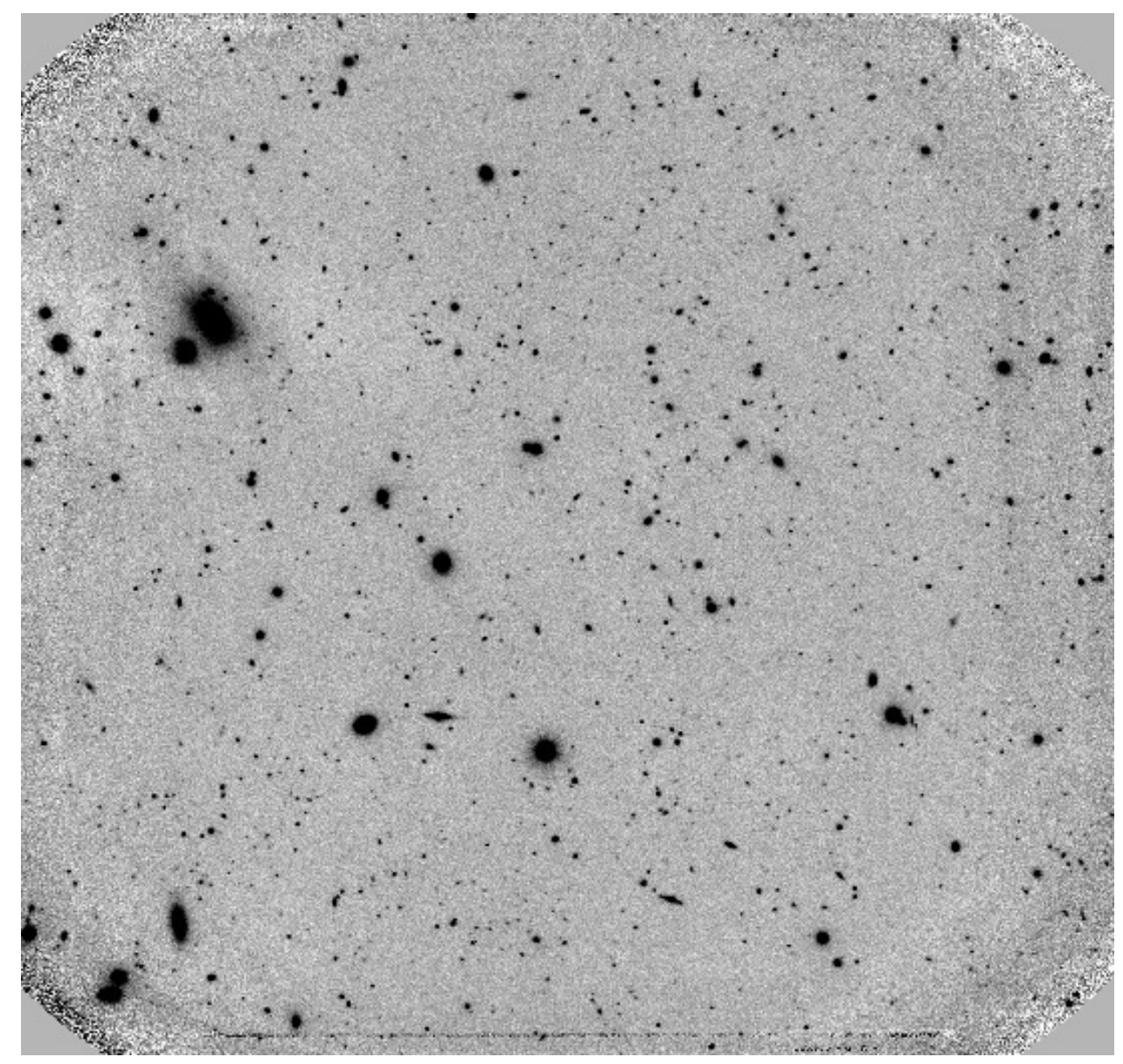

Figure 12. A deep image in Ks-band based on 3600s of integration using a sequence of frames "dithered" in a 9-point box pattern. The field of view of the stacked mosaic is $\sim 6.5$ ' x 6.5 '.

Figure 12 illustrates deep imaging in the $\mathrm{K}_{\mathrm{s}}$-band using a rectangular dither pattern to derive a sky flat. The limiting magnitude in this image is $\mathrm{K}_{\mathrm{s}} \sim 23.5$ (Vega; $\mathrm{S} / \mathrm{N} \sim 10$ in a $0.5^{\prime \prime}$ aperture) based on one hour total integration time. The image quality in the stacked image is FWHM $=0.56 "$. This performance is excellent.

\section{CONCLUSIONS}

MOSFIRE is one of the most challenging cryogenic infrared spectrographs ever attempted. The sheer size, mass and complexity have strained our attempts to create a build-to-cost project. High risk areas such as the CSU, the large optics and the bonding technique for mounting lenses were mitigated by attacking these areas early in the project. Cost was also contained by taking advantage of heritage from previous instruments designed for WMKO. Large test chambers were used to test and evaluate individual mechanisms before they were installed into MOSFIRE. In this way the number of cool downs of the large MOSFIRE dewar was kept to a minimum. Measurements on the telescope confirm laboratory measurements and predictions. MOSFIRE is a powerful, high-throughput and sensitive imager and spectrograph. 


\section{ACKNOWLEDGEMENTS}

MOSFIRE was developed by the consortium of the University of California, Los Angeles, California Institute of Technology, University of California, Santa Cruz and the W. M. Keck Observatory. This material is based upon work supported by AURA through the National Science Foundation under AURA Cooperative Agreement AST 0132798, as amended. Funding has also been provided through a generous donation by Gordon and Betty Moore. It is pleasure to acknowledge the cooperation of the Swiss Centre for Electronics and Micro Technology (CSEM) and Teledyne Imaging Sensors. The authors gratefully acknowledge the outstanding support of the entire Keck Observatory staff during

commissioning, especially Marc Kassis, Greg Wirth and Al Honey. We also thank all of the vendors that have worked with us to produce this instrument.

\section{REFERENCES}

[1] Adkins, S.M., Armandroff, T., Lewis, H., Martin, C., McLean, I.S., Rockosi, C. and Wizinowich, P., “Advances in instrumentation at the W. M. Keck Observatory,” Proc. SPIE 7735, 1. (2010).

[2] McLean, I. S., Steidel, C. C., Matthews, K., Epps, H. and Adkins, S. M., et al., "Design and development of MOSFIRE, the Multi-Object Spectrometer For Infra-Red Exploration at the Keck Observatory," Proc. SPIE 7735, 77351E-77351E-12 (2010).

[3] Kulas, K. R., McLean, I. S. and Steidel, C. C., "Performance of the HgCdTe Detector for MOSFIRE, an Imager and Multi-Object Spectrometer for Keck Observatory," Proc. SPIE 8453-63, this conference (2012).

[4] Konidaris, N. P., Aliado, T., Epps, H. W., Fucik, J., Matthews, K., McLean, I. S., Steidel, C. C., Weber, R. W., "MOSFIRE lens bonding, assembly and alignment," Proc. SPIE 8446-118, this conference (2012).

[5] Spanoudakis, P., Giriens, L., Henein, S., Lisowski, L., O’Hare, A., Onillon, E., Schwab, P. and Theurillat, P., "Configurable slit-mask unit of the Multi-Object Spectrometer for Infrared-Red Exploration for the Keck telescope: Integration and Tests," Proc. SPIE 7018, 7018-19 (2008). 